\title{
Telemedicina baseada em evidência - cirurgia do trauma e emergência (TBE-CITE)
}

\author{
Evidence-based telemedicine - trauma \& acute care surgery (EBT-TACS)
}

tCbC facs Gustavo Pereira Fraga, TCBC-SP; Bartolomeu Nascimento Jr. FRCSC faCS²; Sandro Rizoli²

$\mathrm{R}_{\mathrm{sen}}^{\mathrm{e}}$ ecently, the Brazilian Society of Integrated Trauma Care (SBAIT) and the Brazilian College of Surgeons (CBC) through its Journal (Journal of the Brazilian College of Surgeons) have teamed up with professors from the University of Toronto to reproduce an experience that is already going well in Canada; and has everything to be a great success in Brazil: the Journal Clubs meetings via telemedicine. The target audience is comprised of surgeons, fellows, residents, medical students, as well as graduate and post graduate students. At these Journal Clubs, participating services meet to discuss papers on a topic that has been booked in advance.

A huge number of articles are published every day worldwide. It is a challenge for the surgeon with a busy practice to keep themselves up-to-date. Attending to the meetings and having access to the meeting's reports published in this Journal, will allow Brazilian surgeons to quickly be updated on topics discussed by experts who generate evidence-based recommendations. Therefore, this initiative is a way to quick update non-academic surgeons allowing knowledge to be gained, in the hopes to improve the quality of surgery practiced in this country.

The goal of this educational activity, the Evidence -based Telemedicine - Trauma \& Acute Care Surgery (EBTTACS) is to promote the critical appraisal of scientific articles and current literature in order to generate recommendations on important issues to the daily practice of surgeons who work with trauma, acute care surgery and intensive care of the surgical patient. In preparation for the EBT - TACS meetings, participants perform a literature review with the objective to select the most relevant scientific articles for discussion. The analyses of articles are conducted in a standardized fashion, which should evaluate the design of the study, and the limitations and strengths of the article. In addition to the trauma and acute care surgeon, other experts are invited to participate and suggest topics which they have experience with to be discussed. The recommendations and conclusions of the EBT - TACS meetings are published in the Journal of the CBC and thus available to inform Brazilian surgeons. The participation of centers attending the meetings is acknowledged in the published report. The authors of this editorial will be available to assist at all stages of this initiative; including providing presenters with support to the critical analysis of papers, and also with the writing of the manuscripts resulting from the EBT - TACS meetings to be published in Portuguese and English.

The EBT - TACS meetings are held on the last Tuesday of even months except December, when the meeting will be moved to January. The meetings are in Portuguese and lasts 60 minutes. The scheduled time is 1:00 PM - Toronto, which most of the year is 14:00. Brasilia (time changes of one or two more hours due to daylight saving time in both countries are expected). There is no registration fee to participate in the meetings. Centers interested in participating must register online on the SBAIT (secretaria@sbait.org.br) or CBC (revistacbc@cbc.org.br) websites and provide the number of their IP videoconferencing room. Approximately three weeks preceding the date of the meeting those interested will receive PDF files by e-mail with the articles that will be presented and discussed. Ideally, each service should invite their experts on the topic to be discussed, so that the meetings have a high academic level.

At this early stage the connection is being made via the University of Miami, with great support from Dr. Antonio C. Marttos Jr. We are also contacting the team of Telemedicine University Network (RUTH - http://rute.rnp.br) to enable more centers to have access to telemedicine, even in more remote areas in our continental Brazil.

The first meeting of the EBT - TACS was held on January 17th and discussed "Use of tranexamic acid on blood transfusion in trauma." The conclusions of this meeting are published in this issue of this Journal. In February, the topic to be discussed is "Acute Appendicitis: Medical or Surgical Management?".

Currently, our country is advancing in various aspects, particularly in science. The current government's policies prioritize medical emergencies, and the collaboration between the CBC and SBAIT is fundamental to disseminate knowledge with the intent to better prepare surgeons for the Brazilian population. The EBT - TACS initiative is an opportunity to involve surgical services in several states; exchanging experiences; promoting critical interpretation of current literature; stimulating the production of scientific articles; and ultimately providing education for social development.

1. Universidade Estadual de Campinas / University of Campinas; 2. Universidade de Toronto / University of Toronto. 